Environ. Eng. Res. Vol. 8, No. 3, pp. 122 129, 2003

Korean Society of Environmental Engineers

\title{
FINITE-ELEMENT MODELING ON THE CONVECTIVE TRANSPORT OF AN ORGANIC SOLVENT THROUGH NONUNIFORM SOILS DURING IN-SITU PHOTOLYSIS PROCESS
}

\author{
Kun Sang Lee ${ }^{\dagger}$ \\ Division of Civil and Environmental Engineering \\ Kyonggi University, Suwon, Kyonggi 442-760, Korea \\ (received February 2003, accepted April 2003)
}

\begin{abstract}
In-situ photolysis of a nonuniform soil containing 2,3,7,8-tetrachlorodibenzo-p-dioxin (TCDD) has been theoretically analyzed to develop a numerical model and gain further insight for the physical process involved. This study focuses on the mathematical description and the development of a finite-element mode! for the convective upward transport of an organic solvent driven by photodecomposition at the surface. The model includes the effects of size and its distribution of soil particles, physicochemical characteristics between soil surface and solvent, and initial saturation on the transport velocities through the unsaturated soil zone.

Extensive numerical calculations were performed to yield the profiles of saturation in the simulated nonuniform porous media. For uniform media, larger value of initial saturation results in larger amount of evaporation at the surface. The effect of pore size is almost negligible. For nonuniform media, the effects of nonuniformity become significant as pore size and initial saturation become smaller. Larger initial saturation and smaller pore size lead to a larger variation of saturation with the lowest value at the surface. The simulation studies conducted in this study have shown that the developed model is very useful in analyzing the effects of physical and chemical characteristics on the decontamination process in the soil environments.
\end{abstract}

Key Words : 2,3,7,8-tetrachlorodibenzo-p-dioxin (TCDD), convective transport, finite-element model, nonuniform soil, photolysis,

\section{INTRODUCTION}

The contamination of soil with 2,3,7,8-tetrachlorodibenzo-p-dioxin (TCDD) has become a matter of concern recent years. TCDD has extremely low water solubility and volatility and thus can be very persistent once introduced into the soil environment. Other chemicals such as polychlorinated biphenyls (PCBs) and chlorinated furans exhibit similar behavior. Exposure to high

\footnotetext{
${ }^{\dagger}$ Corresponding author

E-mail: kslee@kyonggi.ac.kr

Tel: +82-31-249-9738, Fax: +82-31-244-6300
}

levels of TCDD can promote chromosome damage, heart attacks, and cancer. Highemperature incineration and chemical treatment have been effective means of soil decontamination. Both of these methods can be very effective, but can also prove to be very expensive.

One of the most promising ways to clean up soil contaminated with TCDD takes advantage of a property of the compound that was discovered recently. Although generally a very stable molecule, TCDD was found to undergo photolysis by sunlight under the proper conditions. This 
discovery led to the possibility of spraying the soil surface with some low-toxicity organic solvent mixtures and allowing time for TCDD solubilization, transport, and photodegradation. The solarinduced photolytic reaction can be a principal mechanism for transforming this chemical to less toxic degradation products.

Dougherty et al. ${ }^{1)}$ performed experimental and numerical studies on the migration of a solvent through uniform media during in-situ decontamination of soils. Their research has demonstrated that a convective upward movement of TCDD subsequent to removing the chemical from the soil particles was found to be the major transport mechanism. A series of wind tunnel tests with uniform or simple nonuniform media were carried out to investigate effects of soil particles on the evaporation in soil. ${ }^{2,3)}$ Most of previous studies, however, derived conclusions based on the experimental results under limited ranges of parameters. Actual migration of a solvent can be affected by chemical properties of soil surfaces as well as physical characteristics such as porosity and saturation. Especially, effective permeability and capillary pressure are functions of saturation. To understand and predict the migration process and saturation profile more effectively, it is necessary to have mechanistic description and numerical model of solvent transport in soil.

This study focuses on the mathematical description of the solvent migration process and the development of a numerical model. With only a limited number of parameters, the developed model allows to represent the effects of size distribution in soil particles, characteristics between soil surface and solvent, and initial saturation on the migration velocities through a nonuniform unsaturated zone. To achieve these goals, the model incorporates a multiphase fluid flow equation in a porous medium and correlation equations on the absolute permeability, relative permeability, and capillary pressure. The resulting nonlinear partial differential equation was solved by a finite-element method. Results of theoretical and numerical studies can provide useful information on the convective transport of a solvent in the unsaturated zone that is characterized by various soil environments.

\section{MATHEMATICAL FORMULATION}

To understand the physical process involved in liquid flow through unsaturated soil zone, a theoretical analysis has been undertaken. This involved deriving and solving a partial differential equation describing the liquid velocity and saturation in the nonuniform soil column.

One major assumption in writing the equation is that the fluid behavior in the soil column can be adequately represented by including only two fluid phases in the model. When the solvent is applied to soil that has been partially saturated with water, there are actually three phases, a gas phase, an aqueous phase, and an organic solvent phase. To avoid complexity, the model described here, however, includes only a wetting liquid phase and a nonwetting vapor phase. Using one of several correlations found in the literature, capillary pressure and relative permeability as functions of saturation are then relatively easy to estimate. Also, the effects of a thermal gradient in the soil environment were not taken into account by use of an energy balance.

\section{Flow Equation}

To solve for saturation and velocity profile, a one-dimensional continuity equation for a wetting fluid can be written as follows:

$$
n \frac{\partial S}{\partial t}=-\frac{\partial}{\partial x}(n S v)
$$

where $n$ is porosity $\left[\mathrm{L}^{3} / \mathrm{L}^{3}\right], S$ is the saturation $\left[\mathrm{L}^{3} / \mathrm{L}^{3}\right]$, and $v$ is the interstitial velocity $\left[\mathrm{LT}^{-1}\right.$, $\mathrm{m} / \mathrm{sec}$ ] of a wetting liquid-phase fluid.

Momentum balance for fluid flow in a porous medium leads to a generalized form of Darcy's law. For multiphase flow, the law relates the velocity of wetting fluid to the effective permeability and pressure gradient. 


$$
n S v=-\frac{k_{e}}{\mu} \frac{\partial p}{\partial x}
$$

where $k_{e}$ is the effective permeability $\left[\mathrm{L}^{2}, \mathrm{~m}^{2}\right]$, $\mu$ the viscosity $\left[\mathrm{ML}^{-1} \mathrm{~T}^{-1}, \mathrm{~Pa} \cdot \mathrm{sec}\right]$, and $p$ the pressure $\left[\mathrm{ML}^{-1} \mathrm{~T}^{-2}, \mathrm{~Pa}\right]$ of a wetting fluid. The effective permeability can be defined the product of the absolute or intrinsic permeability $(k)$ of the porous medium and a relative permeability $\left(k_{r}\right)$ to account for the effect of changing saturation level.

The effect of gravity has been considered to be negligible in this analysis. The pressure of a nonwetting fluid, $p_{n w}$, is zero since it was assumed to be a vapor earlier. Taking into account the fact that $p_{c}=p_{n w}-p$ and $p=-p_{c}$ as a result of previous assumption, one can derive

$$
n S v=\frac{k k_{r}}{\mu} \frac{\partial p_{c}}{\partial x}
$$

where $p_{c}$ is capillary pressure.

By combining Eq. 1 and 3, a one-dimensional equation describing liquid flow equation through an unsaturated soil zone can be derived as follows:

$$
n \frac{\partial S}{\partial t}=-\frac{\partial}{\partial x}\left(\frac{k k_{r}}{\mu} \frac{\partial p_{c}}{\partial x}\right)
$$

The use of Eq. 4 requires knowledge of three soil hydraulic functions: relative permeability curve $k_{r}(S)$, capillary pressure curve $p_{c}(S)$, and intrinsic or absolute permeability $k$. Accurate measurements of these parameters are generally cumbersome, costly, and very time-consuming. Consequently, many attempts have been made to develop indirect methods which predict the function from empirical relationships.

Several correlation equations exist to describe the variation of relative permeability with saturation. A comprehensive review of the different models is given by Assouline. ${ }^{4)}$ The correlation used in this study was the power-law function initially suggested by Brooks and Corey ${ }^{5}$
$k_{r}=\left(\frac{S-S_{r}}{1-S_{r}}\right)^{\frac{2+3 \lambda}{\lambda}}$

where $S_{r}$ is the irreducible saturation at which the wetting phase is in the form of isolated pendular rings. The Brooks-Corey parameter, $\lambda$, usually lies between 0.2 and 3.0. A very large $\lambda$ describes a single grain size material, while a very small value indicates a highly nonuniform material.

To find the variation of capillary pressure with position, a multiphase Darcy's law can be rewritten in terms of the gradient in saturation.

$$
n S v=\frac{k k_{r}}{\mu} \frac{d p_{c}}{d S} \frac{\partial S}{\partial x}
$$

Similar to relative permeability, a number of empirical relationships have been suggested in the literature for capillary pressure or moisture characteristic curve. Vogel et al. ${ }^{6)}$ presented a summary on the variety of empirical equations describing the soil water curve. In this study, the relationship of capillary pressure to saturation is assumed to be adequately represented also by Brooks and Corey ${ }^{5}$ such that

$p_{c}(S)=p_{d}\left(\frac{S-S_{r}}{1-S_{r}}\right)^{\frac{1}{\lambda}}$

The entry pressure $p_{d}\left[\mathrm{ML}^{-1} \mathrm{~T}^{-2}, \mathrm{~Pa}\right]$ is a parameter that is related to the size of largest pore and interfacial tension between water and soil particles by the following equation.

$p_{d}=\frac{2 \sigma \cos \theta}{r_{\max }}$

where $r_{\max }$ is the radius of largest pore $[\mathrm{L}, \mathrm{m}]$, $\sigma$ is an interfacial tension $\left[\mathrm{MT}^{2}, \mathrm{~kg} / \mathrm{sec}^{2}\right], \theta$ is a contact angle. The gradient of capillary pressure in saturation, therefore, can be expressed as 
$\frac{d p_{c}}{d S}=-\frac{1}{\lambda} \frac{p_{d}}{\left(1-S_{r}\right)^{-\frac{1}{\lambda}}}\left(S-S_{r}\right)^{-\frac{\lambda+1}{\lambda}}$

The correlation equation, which is derived from capillary pressure-saturation curve, was also used to determine the absolute permeability of an unsaturated zone. Several authors including Purcell $^{7)}$, Swanson ${ }^{8)}$, and Thomeer" have proposed correlation equations approximating the absolute permeability with capillary pressure data. Swanson's correlation was adopted in this study because it utilizes only the shape of $p_{c}(S)$ curves without any other parameters describing geometrical characteristics of pore networks. Transformation of the Swanson's correlation, that is derived originally in a British unit system, into a SI unit system results in

$$
k=0.17813\left(\frac{1-S}{P_{C}}\right)_{A}^{2.005}
$$

In the equation, the subscript $A$ represents the point where the ratio $\left(\frac{S}{p_{c}}\right)$ has a maximum value.

Substitutions of Eq. 5, 6, 9, and 10 to Eq. 4 and rearrangement produce a singe flow equation describing saturation and velocity profiles as functions of time and location.

$$
\begin{aligned}
& n \frac{\partial S}{\partial t}=-\frac{\partial}{\partial x}\left[\frac{1}{\mu}\left\{0.17813\left(\frac{1-S}{p_{c}}\right)_{A}^{2.005}\right\}\left(\frac{S-S_{r}}{1-S_{r}}\right)^{\frac{2+3 \lambda}{\lambda}}\right. \\
& \left.\left\{-\frac{1}{\lambda} \frac{p_{d}}{\left(1-S_{r}\right)^{\frac{1}{\lambda}}}\left(S-S_{r}\right)^{\frac{\lambda+1}{\lambda}}\right\} \frac{\partial S}{\partial x}\right]
\end{aligned}
$$

\section{Initial and Boundary Conditions}

The equation above can be solved with appropriate initial and boundary conditions. At the bottom of column, the flux of liquid is set to be zero to represent a situation of a photodegradation experiment.

$$
v=0 \quad \text { at } \quad x=0
$$

At the top of column, the boundary condition used was based on the amount of solvent lost by evaporation. A lot of external environmental conditions and internal soil properties control evaporation in soil. ${ }^{10)}$ In this study, the maximum liquid velocity at the surface $\dot{M}_{0}$ $\left[\mathrm{LT}^{-1}, \mathrm{~m} / \mathrm{sec}\right]$ can be found by relating the rate of degradation $R_{\text {evap }}\left[\mathrm{MT}^{-1}, \mathrm{~kg} / \mathrm{sec}\right]$ to the surface area of the column, $A\left[\mathrm{~L}^{2}\right]$. Moreover, to account the effect of saturation on the evaporation rate, a linear evaporation efficiency is assumed for an unsaturated surface. This boundary condition can be expressed

$$
v=\frac{R_{\text {evap }}}{\rho A}\left(S-S_{r}\right)=\dot{M}_{0}\left(S-S_{r}\right) \text { at } x=l
$$

where $\rho$ is the density of liquid $\left[\mathrm{ML}^{-3}, \mathrm{~kg} / \mathrm{m}^{3}\right]$.

The initial condition used for the solution of the equation was that the liquid saturation was a known constant value at the start of the test.

$$
S=S_{0} \text { at } t=0
$$

\section{RESULTS AND ANALYSIS}

\section{Finite-Element Model}

The equation for saturation and velocity profiles were solved numerically using a onedimensional finite-element model developed in this study. The model was developed with a general Galerkin method. ${ }^{\text {(I) }}$

The numerical calculations simulate the sunlight exposure experiment. Organic solvent evaporates at the top surface from $0.2 \mathrm{~m}$ deep soil column. Constant liquid density $\left(1000 \mathrm{~kg} / \mathrm{m}^{3}\right)$ and viscosity $(0.001 \mathrm{~Pa} \cdot \mathrm{sec})$ are assumed due to an isothermal system and relatively small pressure changes. The porosity and irreducible liquid saturation of the soil column are taken to be 0.4 and 0.05 . The geometrical properties of soil particles consisting in the unsaturated zone are characterized by entry pressure and BrooksCorey parameter which represent largest size of particles or pores and size distribution, respec- 
tively. A number of calculations were carried out for a wide range of soil structure and texture using $\lambda$ values from 0.2 to 3.0 and $p_{d}$ between 50 and $500 \mathrm{kPa}$. Evaporation rate at the ground surface was set to be $1 \times 10^{-7} \mathrm{~m} / \mathrm{sec}$.

The data in Table 1 constitute the absolute permeabilities estimated using Eq. 10, ranging from $2.66 \times 10^{-15} \mathrm{~m}^{2}$ to $1.35 \times 10^{-11} \mathrm{~m}^{2}$ for given parameters. A combination of larger values of Brooks-Corey parameter and smaller values of entry pressure, which represents a uniform medium composed of large particles, results in larger absolute permeabilities.

Table 1. Absolute permeability $\left[\mathrm{m}^{2}\right]$ calculated from Swanson's correlation in terms of Brooks-Corey parameter $(\lambda)$ and entry pressure $\left(p_{d}\right)$

\begin{tabular}{c|c|c|c|c}
\hline$\lambda p_{d}$ & $50 \mathrm{kPa}$ & $100 \mathrm{kPa}$ & $200 \mathrm{kPa}$ & $500 \mathrm{kPa}$ \\
\hline \hline 0.2 & $2.70 \times 10^{-13}$ & $6.72 \times 10^{-14}$ & $1.67 \times 10^{-14}$ & $2.66 \times 10^{-15}$ \\
\hline 0.5 & $1.32 \times 10^{-12}$ & $3.30 \times 10^{-13}$ & $8.22 \times 10^{-14}$ & $1.31 \times 10^{-14}$ \\
\hline 1.0 & $3.78 \times 10^{-12}$ & $9.42 \times 10^{-13}$ & $2.35 \times 10^{-13}$ & $3.74 \times 10^{-14}$ \\
\hline 3.0 & $1.35 \times 10^{-11}$ & $3.37 \times 10^{-12}$ & $8.40 \times 10^{-13}$ & $1.34 \times 10^{-13}$ \\
\hline
\end{tabular}

The relative permeability of a wetting liquid is displayed in Figure 1 in terms of saturation and Brooks-Corey parameter. The larger BrooksCorey parameter, the larger relative permeability becomes. Figure 2 gives changes in capillary pressures when the entry pressure is $50 \mathrm{kPa}$. Much larger values of capillary pressure are

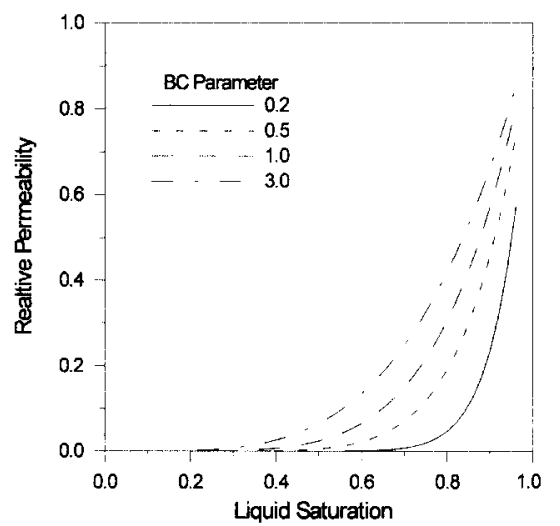

Figure 1. Relative permeability of liquid as a function of saturation calculated from Brooks-Corey correlation.

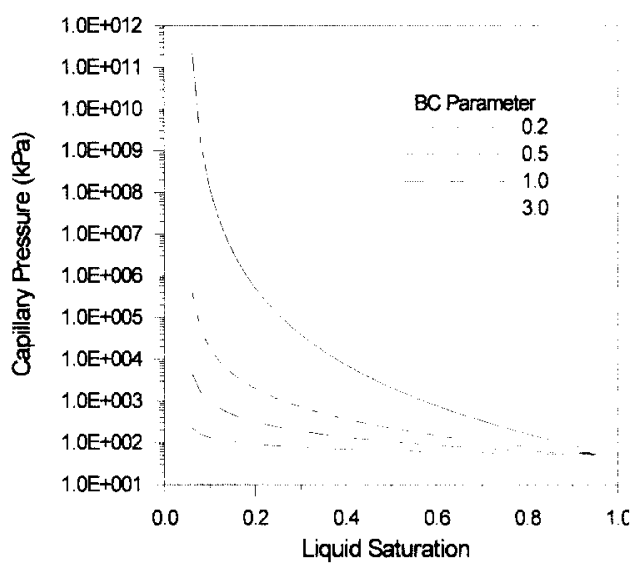

Figure 2. Capillary pressure as a function of liquid saturation calculated from Brooks-Corey correlation $\left(p_{d}=50 \mathrm{kPa}\right)$.

obtained with smaller values of Brooks-Corey parameter. Results from additional calculations for larger entry pressure up to $500 \mathrm{kPa}$ indicate that capillary pressure values become larger due to smaller particle or pore size. However, the overall shape of curves remains essentially unchanged.

\section{Numerical Results and Discussion}

To determine how well the numerical model represented the physical situation, it was applied to a uniform medium chosen as an illustrative example. A simulation was made for a uniform soil column of initial saturation 0.9 exposed to sunlight for $200 \mathrm{hr}$. Given in Figure 3 as the

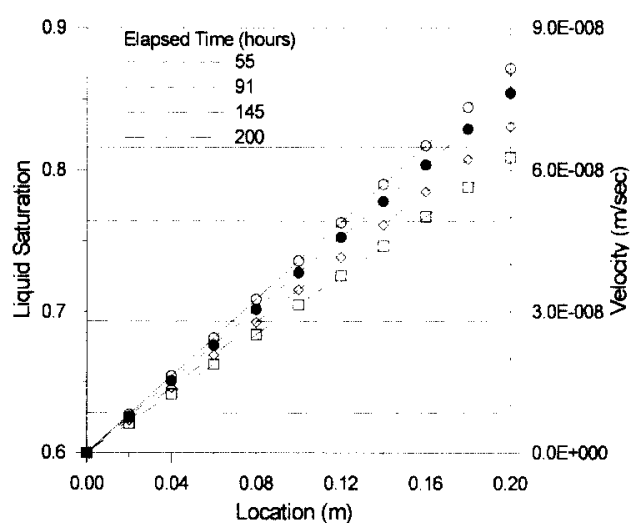

Figure 3. Saturation and velocity profiles for the case of uniform grains with $p_{d}=50 \mathrm{kPa}$ and $S_{0}=0.9$ after $200 \mathrm{hr}$. 
predicted saturation and velocity profiles throughout the soil column, results of these preliminary numerical calculations were compared against results of laboratory and numerical experiments reported in Dougherty et al.'s paper.' Because detailed information on the conditions of experiments was unavailable, the results cannot be directly checked. Overall shapes of liquid saturation and velocity curves for a uniform medium after 200 -hr evaporation were, therefore, examined. The general shapes of the curves are appropriate with little higher saturation at the bottom of the column and almost linearly increasing velocity as approaching the top. As time elapsed, the velocity decreases due to a saturation-dependent boundary condition assigned at the surface. Decreased saturation causes lowering evaporation efficiency and rate.

The effects of changes in pore size and initial saturation on the saturation profiles were further analyzed by numerically simulating the liquid migration through a uniform soil column. Figure 4 illustrates how liquid saturation changes inside the soil column with initial saturation ranging 0.5 to 0.9. Results for two additional cases

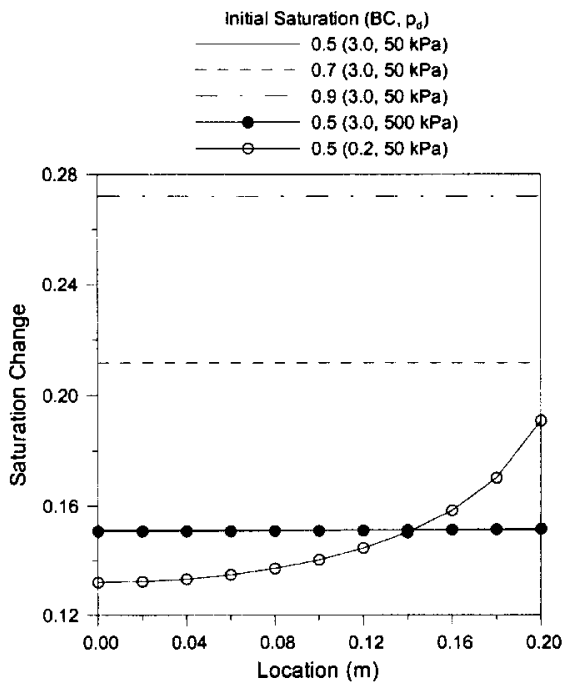

Figure 4. Profiles of saturation changes for the cases of uniform and nonuniform grains as functions of initial saturation and entry pressure after $200 \mathrm{hr}$. involving uniform $\left(\lambda=3.0, p_{d}=500 \mathrm{kPa}\right)$ and highly nonuniform $\left(\lambda=0.2, p_{d}=50 \mathrm{kPa}\right)$ media are also presented to examine the effects of pore size and nonuniformity. Higher initial saturation results in larger amount of evaporation mainly due to larger relative permeability and higher evaporation efficiency at the ground surface. For uniform media, values of the saturation difference curves are little small at the bottom of the column. Comparison of saturation changes in uniform $\left(\lambda=3.0, S_{o}=0.5, \quad p_{d}=50 \mathrm{kPa}\right)$ and nonuniform media $\left(\lambda=0.2, \quad S_{o}=0.5, \quad p_{d}=50\right.$ $\mathrm{kPa}$ ) reveals that much larger variation is obtained from a nonuniform medium with the highest evaporation at the surface. Insignificant differences are observed between results from cases of entry pressures of 50 and $500 \mathrm{kPa}$. The observation means that the effects of pore size

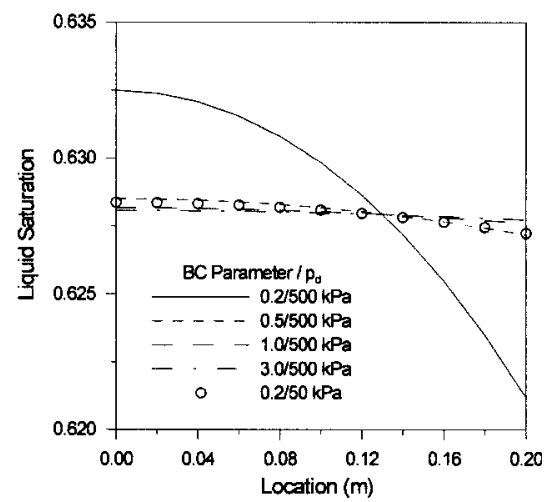

(a) $\mathrm{S}_{\mathrm{o}}=0.9$

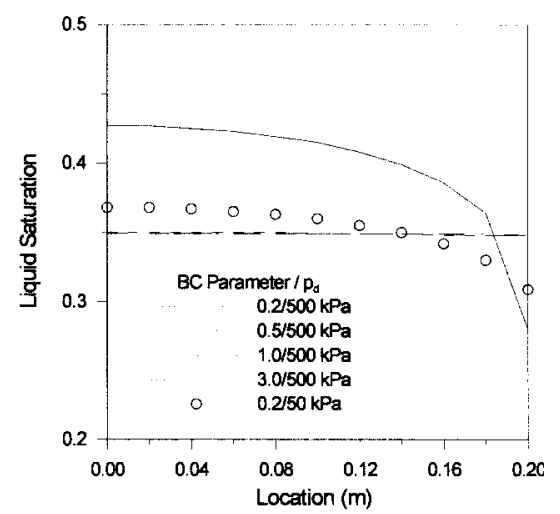

(b) $\mathrm{S}_{\mathrm{o}}=0.5$

Figure 5. Saturation profiles for the cases of nonunifrom grains with different sizes after $200 \mathrm{hr}$. 
are almost negligible for a uniform soil.

Calculations were performed to investigate the effects of nonuniformity of soil columns on the liquid migration. The liquid saturation was calculated after $200 \mathrm{hr}$ for the cases of an initial saturation ranging from 0.5 to 0.9 for entry pressures of 50 and $500 \mathrm{kPa}$. Figures 5(a) and (b) present results obtained with different levels of $S_{o}, p_{d,}$ and $\lambda$. We compare five different scenarios for each saturation level.

As Brooks-Corey parameter approaches to 0.2, the saturation near the surface reduces rapidly for all cases considered. This trend becomes much clearer as the initial saturation becomes smaller and entry pressure larger. For the case of initial saturation of 0.9 , the difference in saturation distribution was almost negligible for a uniform medium and less than $2 \%$ even for highly nonuniform media $(\lambda=0.2$ ). Reduction of initial saturation to 0.5 increased a variation dramatically in the profiles of saturation difference up to $50 \%$. Saturation differences are 0.001 for $S_{o}=0.9$ and 0.06 for $S_{o}=0.5$ in highly nonuniform coarse particles represented by smaller $p_{d}$. For the case of $\lambda=0.2$ and $p_{d}=500$ $\mathrm{kPa}$, the differences increase up to 0.011 for $S_{0}$ $=0.9$ and 0.14 for $S_{0}=0.5$. In summary, the effects of nonuniformity on the saturation variation increase as pore size and initial saturation become smaller.

\section{CONCLUSIONS}

During in-situ cleanup of TCDD $(2,3,7,8$ tetrachlorodibenzo-p-dioxin) contaminated soil, a convective transport of an organic solvent is very important to the maximization of TCDD loss. Based on the mathematical analysis, a finite-element model was developed for the convective upward movement of an organic solvent through a nonuniform unsaturated zone. With Brooks-Corey parameter $\lambda$ and entry pressure $p_{d}$, the mathematical formulation incorporates various correlation equations for relative permeability, capillary pressure, and nonuniformity into continuity equation and Darcy's law of multi- phase flow. The developed finite-element model is, therefore, capable of predicting the effects of pore size distribution, absolute permeability, entry pressure, and liquid saturation. Results of numerical simulations on the flow through uniform media indicate that higher initial saturation increases the amount of evaporation. In the nonuniform media, the effects of nonuniformity become larger as pore size and initial saturation become smaller. Larger initial saturation and smaller pore size lead to a remarkably larger velocity near the surface. The numerical simulation studies conducted in this study have shown that the model provides a useful tool for analyzing the effects of physical and chemical characteristics on the convective migration process of a solvent in the soil environments.

\section{REFERENCES}

1. Dougherty, E.J., McPeters A.L., Overcast M.R., and Carbonell, R.G., "Theoretical analysis of a method for in situ decontamination of soil containing 2,3,7,8-tetrachlorodibenzo-p-dioxin," Environ. Sci. Technol., 27, 505-515 (1993).

2. Lee, S.-D. and Chung, I.-H., "Study on evaporation from the soil affected by the particle size and soil water content," J. Kor. Soc. Env. Eng., 19, 1 - 8 (1997).

3. Lee, S.-D., Park, S.-C., and Lee, K.-I., “A study on the latent heat exchange model between soil and atmosphere," J. Kor. Soc. Env. Eng., 20, 1511 1522 (1998).

4. Assouline, S., "A model for soil relative hydraulic conductivity based on the water retention characteristic curve," Water Resour. Res., 37, 265 271 (2001).

5. Helmig, R., Multiphase Flow and Transport Processes in the Subsurface, Springer, Berlin, Germany, pp. 47 82 (1997).

6. Vogel, T., van Genuchten, M. Th., and Cislerova, M., "Effect of the shape of the soil hydraulic functions near saturation on variably-saturated predictions," $A d v$. in Water Resourses, 24, 505-521 (2000).

7. Purcell, W.R., "Capillary pressures-their mea- 
surement using mercury and the calculation of permeability therefrom," Trans. AIME, 186, $39 \sim 48$ (1949).

8. Swanson, B.F., "A simple correlation between permeabilities and mercury capillary pressures," J. Pet. Technol., 2498 2504 (1981).

9. Thomeer, J.H., "Air permeability as a func- tion of three pore-network parameters," $J$. Pet. Technol., 809 -813 (1983).

10. Yang, M. and Yanful, E.K., "Water balance during evaporation and drainage in cover soils under different water table conditions," Adv. in Environ. Res., 6, 505 521 (2002).

11. Sewell, G., Analysis of a Finite Element Method, Springer-Verlag, New York (1985). 